\title{
Selective approach to efficient ethanol production using adaptation of producer Saccharomyces cerevisiae to hyperosmotic medium
}

\author{
Yelena A. Oleinikova, Tatyana V. Kuznetsova, Margarita G. Saubenova, Aida \\ A. Aitzhanova, Marzhan M. Shormanova, Laura T. Raimbekova \\ Laboratory of Physiology and Biochemistry of Microorganisms, RSOE "Institute of Microbiology and \\ Virology" CS MES RK, Republic of Kazakhstan
}

\begin{abstract}
The resistance to ethanol of 18 strains of alcoholic yeast Saccharomyces cerevisiae, including production strains, was studied. It is shown that yeast growth was inhibited by increasing the concentration of ethanol in the medium above $7 \%(\mathrm{v} / \mathrm{v})$. Successive adaptation of alcohol yeast to gradually increased concentrations of sodium chloride in the medium was carried out. Yeast variants that exceeded production strains in fermentation activity on the medium with $10 \%(\mathrm{v} / \mathrm{v})$ ethanol were received. The use of selected strains will increase the productivity of the process of ethyl alcohol production at the same costs.
\end{abstract}

Keywords: adaptation, ethanol production, hyperosmotic pressure, tolerance, yeast.

\section{INTRODUCTION}

The production of bioethanol is of great interest in connection with the need for renewable energy sources and reducing the negative impact of exhaust gases on the environment. Biodiesel and bioethanol are considered as the main renewable biofuels $[1,2]$. Even a very small addition of ethanol to the fuel used for internal combustion engines significantly saves energy and reduces the toxicity of exhaust gases. Currently, more than half of world production of ethanol is used as an additive to gasoline and only $15 \%$ for the production of alcoholic beverages [3]. At the same time, bioethanol fuel has the greatest potential in comparison with biodiesel, since it is characterized by inexhaustible sources of production. These can be different vegetable raw materials, as well as agricultural and wood-processing waste.

The main direction of the development of alcohol industry is the search for ways to reduce the production costs of raw materials and energy resources. One of the most effective ways of solving this problem is using the technology of fermentation of highly concentrated wort. Increase in productivity in ethanol production is achieved both by improving the technology and increasing the efficiency of the microbiological process [4-6]. Among technological solutions, much attention is paid in recent years to the immobilization of yeast cells on various carriers [6-9], which allows the use of bioethanol producer culture up to four or even seven cycles.

Nevertheless, the periodicity of the process of alcohol fermentation leads to its termination after accumulation of about $8 \%$ ethanol because of its
High toxicity for yeast. Nevertheless, the periodicity of the process of alcohol fermentation necessitates the resumption of fermentation after accumulation of about $8-10 \%$ ethanol because of its high toxicity for yeast. Therefore, the most important aspect of intensifying the microbiological industry is to increase the resistance of the producer strain to the final fermentation product ethanol [10-14].

In the process of alcohol production, yeast cells are exposed to the effects of hyperosmotic and ethanol stress. Adaptation of cells in the conditions of damage occurs not only at the metabolic and functional levels. Long, repeated or significant damage leads to essential structural rearrangements in a cell that have an adaptive value [15-17]. Such adaptation to the action of damaging factors occurs through stereotyped adaptive changes in the cell or cellular system. It is known that the membrane fluidity of the cells is of paramount importance in nonspecific resistance to abiotic factors of the environment.

The purpose of this work was to increase production characteristics, firstly ethanol resistance, of alcoholic yeast Saccharomyces cerevisiae by adapting to the hyperosmotic pressure of the environment.

\section{MATERIALS AND METHODS}

Alcoholic yeast Saccharomyces cerevisiae (18 strains, including two production strains No.13 and No.18) were the subjects of investigation.

Alcohol resistance of the yeast cultures was determined in the liquid Reader medium ( $\mathrm{g}$ per L: glucose - 10.0, $\left(\mathrm{NH}_{4}\right)_{2} \mathrm{SO}_{4}-2.1, \mathrm{KH}_{2} \mathrm{PO}_{4}-1.0$, $\mathrm{MgSO}_{4}-0.2, \mathrm{~K}_{2} \mathrm{SO}_{4}-0.2$, yeast extract -0.5 ). Yeast was inoculated into the flask with an alcohol 
content of $1 \%$ to $15 \%(\mathrm{v} / \mathrm{v})$ at intervals of $1 \%$. Five $\mathrm{ml}$ of pure yeast culture $\left(10^{8}\right.$ colony forming units CFU per ml) was added to a flask with $100 \mathrm{ml}$ of the medium. The flasks were incubated for $120 \mathrm{~h}$ at $30^{\circ} \mathrm{C}$ under conditions of a batch culture. Reader medium with an alcohol content of 1 to $15 \%(\mathrm{v} / \mathrm{v})$ was used as a control. Samples of yeast suspensions $(4 \mathrm{ml})$ for determining the optical density $\mathrm{D}$ (with a green filter and wavelength $\lambda=540 \mathrm{~nm}$ ) were taken immediately after inoculation and after 24 and 120 hours.

To increase osmotolerance, alcohol yeast was grown on a liquid Rider medium with gradually increasing concentrations of sodium chloride from $5 \%$ to $15 \%$ (with an increase in the concentration of sodium chloride by $1 \%$ ). Five $\mathrm{ml}$ pure yeast culture $\left(10^{8} \mathrm{CFU} / \mathrm{ml}\right)$ was added to a flask with $100 \mathrm{ml}$ of the medium. The incubation was carried out under conditions of a batch culture for $72 \mathrm{~h}$ at $30^{\circ} \mathrm{C}$. Samples for determining the optical density $\mathrm{D}$ of the yeast suspension $(\lambda=540 \mathrm{~nm})$ and CFU quantity (on Reader medium) were taken immediately after inoculation and after $72 \mathrm{~h}$ incubation. $72 \mathrm{~h}$ yeast suspensions were used for inoculation into a medium with a higher concentration of $\mathrm{NaCl}$.

The fermentation activity of the selected and initial variants was examined in Dunbar tubes on filtered wheat wort with a solids concentration of $16.4 \%$ and $\mathrm{pH} 4.3,8 \%(\mathrm{v} / \mathrm{v})$ or $10 \%(\mathrm{v} / \mathrm{v})$ of ethanol was added. The inoculum cultures $\left(10^{8} \mathrm{KOE} / \mathrm{ml}\right)$ in an amount of $2 \mathrm{ml}$ were inoculated into tubes with $20 \mathrm{ml}$ of the medium and cultivated for 24 hours at $28^{\circ} \mathrm{C}$. The intensity of fermentation was judged by the amount of the displaced culture medium at the sealed end of the tube, which was released during the fermentation by carbon dioxide.

All experiments were carried out in triplicate. The results of the studies were statistically processed according to a standard procedure using the Student's test.

\section{RESULTS AND DISCUSSION}

Tolerance of 18 strains of alcohol yeast to the content of ethanol in the media from 1 to $10 \%$ was investigated. It was noted that yeast growth was largely inhibited at concentrations of ethanol of $7 \%$ and above. Figures 1 and 2 show data on the accumulation of biomass by yeast Saccharomyces cerevisiae in the media with $7 \%$ and $10 \%$ ethyl alcohol.

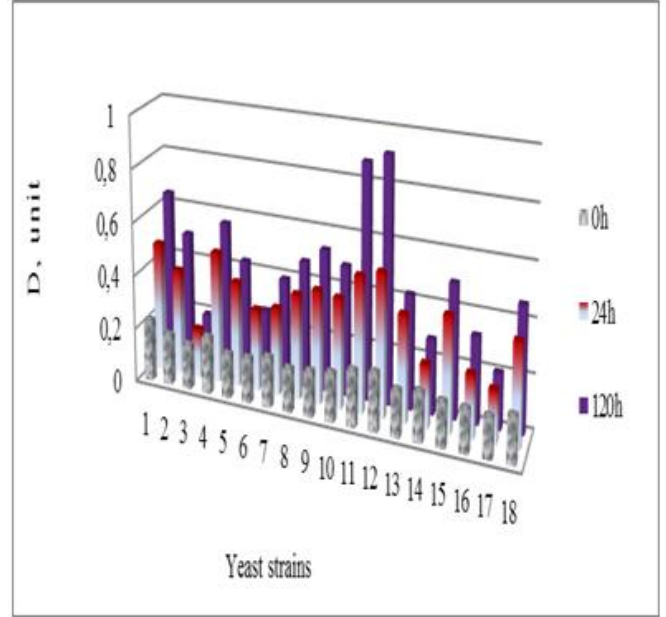

Figure1: The increase in the biomass of alcohol yeast in the culture medium with $7 \%$ ethanol

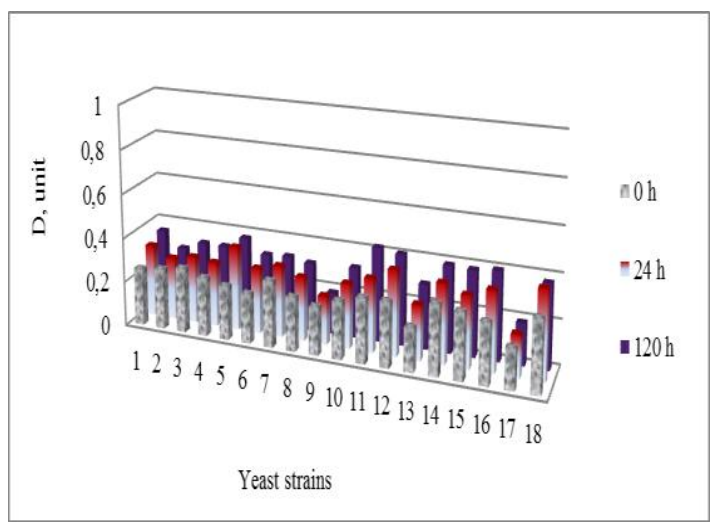

Figure2: The increase in the biomass of alcohol yeast in the culture medium with $10 \%$ ethanol

The bulk of the strains studied showed the ability to grow in a medium with $7 \%$ ethanol content, although the growth of a number of cultures was largely inhibited. Growth of yeast at $10 \%$ ethanol content was lean or absent.

The yeast cultures under investigation were adapted to the gradually increasing concentration of sodium chloride.

It was shown that the studied strains of alcohol yeast differed in their resistance to increased salinity of the environment and had a different adaptive potential. Thus, as shown in Table 1 some strains (No. 1, 5, 6, 12) were able to grow in media with 5-7\% NaCl, and strains No. 2, 3, 16, and 17 withstood even higher concentrations of $\mathrm{NaCl}$. About a half of the strains were characterized by poor growth in the media with addition of sodium chloride.

The viability of yeast cells was noted to drop starting with the concentration of sodium chloride in the medium of $9 \%$ - Table 2 . The number of viable cells in $1 \mathrm{ml}$ decreased in nine out of 18 
strains by $5-87 \%$ with a slight increase in the optical density of the suspensions. However, some cultures retained the capacity for active growth.

Viability was reduced only in six cultures (by $14-85 \%$ ) when $\mathrm{NaCl}$ concentration was increased in the medium up to $11 \%$, since the material for inoculation was taken each time from the previous incubation, and thus more tolerant variants were selected with every inoculation. In five yeast cultures the CFU number increased 17.0-60.0 times, in seven ones it did 1.6-7.8 times.

Viability inhibition was more pronounced in medium with $13 \%$ and $15 \%$ sodium chloride. At $13 \% \mathrm{NaCl}$ it decreased by $24 \%$ in two cultures and by $70-80 \%$ in seven cultures. At the same time, a significant increase in CFU (7.9-18.5 times) was noted in three cultures and slight rise (1.3-5.0 times) - in five cultures.

CFU quantity decreased in 17 cultures by $17-82 \%$ and increased by 1.1-2.2 times in 8 cultures in the medium with the maximum $\mathrm{NaCl}$ content.
Only cultures No.7 and No.15 differed in ability not only withstand the concentration of sodium chloride $15 \%$ but even grow in a hyperosmotic environment. So, in culture No. 7, CFU increased 9.2 times, in culture No. 15 - 3.5 times, the optical density of culture suspensions rose correspondingly by 1.9 and 1.4 times.

Based on the data obtained, cultures No. 7 and No. 15 which were adapted to the high $\mathrm{NaCl}$ content and characterized by altered fluidity of the cell membrane and increased tolerance to the effects of abiotic stress factors were used for the selection of variants. The fermentative activity of the selected variants was compared in Dunbar tubes with the activity of the production strain of alcohol yeast. Fermentation of the production strain was stopped at $8 \%$ ethanol in the medium. The selected variants were characterized by high fermentative activity in the absence of $\mathrm{CO}_{2}$ release by the production strain of alcohol yeast after 24 hours of cultivation in the wort with $10 \%$ (v/v) ethyl alcohol - Figure 3.

Table 1: Optical density (units) of alcohol yeast cultures in media with $5-15 \% \mathrm{NaCl}$

\begin{tabular}{|c|c|c|c|c|c|c|c|c|c|c|c|c|}
\hline & \multicolumn{12}{|c|}{$\mathrm{NaCl}$ concentration } \\
\hline No. & \multicolumn{2}{|c|}{$5 \%$} & \multicolumn{2}{|c|}{$7 \%$} & \multicolumn{2}{|c|}{$9 \%$} & \multicolumn{2}{|c|}{$11 \%$} & \multicolumn{2}{|c|}{$13 \%$} & \multicolumn{2}{|c|}{$15 \%$} \\
\hline & $0 \mathrm{~h}$ & $96 \mathrm{~h}$ & $0 \mathrm{~h}$ & $96 \mathrm{~h}$ & $0 \mathrm{~h}$ & $96 \mathrm{~h}$ & $0 \mathrm{~h}$ & $96 \mathrm{~h}$ & $0 \mathrm{~h}$ & $96 \mathrm{~h}$ & $0 \mathrm{~h}$ & $96 \mathrm{~h}$ \\
\hline 1 & 0.381 & 0.896 & 0.449 & 0.823 & 0.502 & 0.632 & 0.359 & 0.603 & 0.426 & 0.500 & 0.426 & 0.598 \\
\hline 2 & 0.407 & 0.745 & 0.431 & 0.729 & 0.518 & 0.699 & 0.504 & 0.681 & 0.428 & 0.643 & 0.428 & 0.582 \\
\hline 3 & 0.410 & 0.706 & 0.412 & 0.721 & 0.440 & 0.755 & 0.353 & 0.730 & 0.395 & 0.695 & 0.395 & 0.567 \\
\hline 4 & 0.384 & 0.710 & 0.403 & 0.621 & 0.392 & 0.539 & 0.358 & 0.503 & 0.374 & 0.489 & 0.374 & 0.450 \\
\hline 5 & 0.387 & 0.739 & 0.427 & 0.684 & 0.486 & 0.647 & 0.545 & 0.590 & 0.431 & 0.508 & 0.431 & 0.454 \\
\hline 6 & 0.382 & 0.747 & 0.550 & 0.679 & 0.402 & 0.584 & 0.493 & 0.535 & 0.475 & 0.529 & 0.475 & 0.521 \\
\hline 7 & 0.408 & 0.875 & 0.431 & 0.869 & 0.405 & 0.851 & 0.473 & 0.803 & 0.402 & 0.786 & 0.402 & 0.747 \\
\hline 8 & 0.402 & 0.816 & 0.402 & 0.792 & 0.362 & 0.695 & 0.354 & 0.631 & 0.493 & 0.574 & 0.493 & 0.549 \\
\hline 9 & 0.404 & 0.548 & 0.407 & 0.545 & 0.371 & 0.540 & 0.384 & 0.485 & 0.367 & 0.449 & 0.367 & 0.413 \\
\hline 10 & 0.397 & 0.664 & 0.401 & 0.602 & 0.398 & 0.562 & 0.359 & 0.531 & 0.420 & 0.509 & 0.420 & 0.491 \\
\hline 11 & 0.430 & 0.678 & 0.428 & 0.675 & 0.534 & 0.672 & 0.374 & 0.632 & 0.510 & 0.617 & 0.510 & 0.594 \\
\hline 12 & 0.409 & 0.788 & 0.390 & 0.591 & 0.420 & 0.560 & 0.366 & 0.520 & 0.433 & 0.742 & 0.433 & 0.451 \\
\hline 13 & 0.409 & 0.583 & 0.403 & 0.564 & 0.365 & 0.537 & 0.455 & 0.521 & 0.478 & 0.512 & 0.478 & 0.500 \\
\hline 14 & 0.384 & 0.559 & 0.416 & 0.554 & 0.382 & 0.550 & 0.474 & 0.540 & 0.386 & 0.534 & 0.346 & 0.527 \\
\hline 15 & 0.404 & 0.765 & 0.423 & 0.741 & 0.395 & 0.734 & 0.513 & 0.710 & 0.496 & 0.701 & 0.496 & 0.686 \\
\hline 16 & 0.386 & 0.858 & 0.430 & 0.820 & 0.404 & 0.750 & 0.480 & 0.698 & 0.394 & 0.607 & 0.394 & 0.548 \\
\hline 17 & 0.382 & 0.789 & 0.363 & 0.610 & 0.397 & 0.556 & 0.340 & 0.703 & 0.516 & 0.672 & 0.516 & 0.627 \\
\hline 18 & 0.381 & 0.622 & 0.364 & 0.614 & 0.374 & 0.598 & 0.345 & 0.582 & 0.391 & 0.579 & 0.391 & 0.573 \\
\hline $\mathrm{K}$ & 0.357 & 0.357 & 0.357 & 0.357 & 0.358 & 0.358 & 0.359 & 0.359 & 0.360 & 0.360 & 0.361 & 0.361 \\
\hline & & & & & & & & . & & & & \\
\hline
\end{tabular}

Table 2: Number of colony forming units $\left(\times 10^{8} / \mathrm{ml}\right)$ in media with $5-15 \% \mathrm{NaCl}$

\begin{tabular}{|c|c|c|c|c|c|c|c|c|c|c|c|c|}
\hline & $5 \%$ & & $7 \%$ & & $9 \%$ & & $11 \%$ & & $13 \%$ & & $15 \%$ & \\
\hline No. & $0 \mathrm{~h}$ & $96 \mathrm{~h}$ & $0 \mathrm{~h}$ & $96 \mathrm{~h}$ & $0 \mathrm{~h}$ & $96 \mathrm{~h}$ & $0 \mathrm{~h}$ & $96 \mathrm{~h}$ & $0 \mathrm{~h}$ & $96 \mathrm{~h}$ & $0 \mathrm{~h}$ & $96 \mathrm{~h}$ \\
\hline 1 & 2.3 & 820.0 & 7.1 & 210.0 & 8.2 & 6.7 & 1.4 & 9.4 & 4.6 & 0.9 & 4.2 & 9.4 \\
\hline 2 & 5.3 & 40.0 & 6.7 & 94.0 & 8.5 & 14.0 & 4.3 & 23.0 & 4.8 & 8.5 & 4.3 & 7.0 \\
\hline 3 & 5.8 & 24.0 & 6.0 & 92.0 & 6.4 & 78.0 & 1.2 & 72.0 & 4.0 & 20.0 & 3.8 & 5.4 \\
\hline 4 & 2.7 & 29.0 & 4.9 & 22.0 & 6.9 & 0.9 & 1.3 & 0.2 & 3.1 & 0.8 & 3.1 & 0.9 \\
\hline 5 & 3.1 & 32.0 & 6.6 & 86.0 & 3.2 & 7.1 & 7.1 & 6.1 & 4.9 & 1.2 & 4.7 & 0.9 \\
\hline 6 & 2.5 & 41.0 & 7.5 & 84.0 & 4.6 & 2.8 & 8.2 & 4.3 & 5.7 & 2.2 & 5.1 & 3.2 \\
\hline 7 & 5.4 & 640.0 & 6.8 & 580.0 & 4.7 & 290.0 & 6.9 & 98.0 & 4.0 & 74.0 & 3.7 & 34.0 \\
\hline
\end{tabular}




\begin{tabular}{|c|c|c|c|c|c|c|c|c|c|c|c|c|}
\hline 8 & 4.8 & 310.0 & 4.7 & 110.0 & 1.9 & 9.3 & 1.7 & 32.0 & 5.5 & 4.2 & 5.9 & 4.9 \\
\hline 9 & 5.0 & 1.6 & 5.3 & 1.2 & 2.0 & 0.9 & 2.1 & 0.9 & 2.8 & 0.8 & 3.9 & 0.7 \\
\hline 10 & 4.3 & 1.0 & 4.6 & 9.7 & 4.0 & 1.8 & 1.9 & 4.0 & 4.6 & 1.4 & 4.1 & 1.3 \\
\hline 11 & 6.7 & 16.0 & 6.7 & 78.0 & 8.5 & 8.1 & 2.0 & 34.0 & 6.5 & 6.8 & 8.0 & 8.9 \\
\hline 12 & 5.6 & 79.0 & 6.5 & 8.1 & 6.1 & 1.4 & 1.9 & 3.1 & 5.0 & 59.0 & 4.6 & 0.8 \\
\hline 13 & 5.7 & 9.1 & 5.0 & 2.8 & 2.0 & 0.9 & 6.4 & 3.2 & 5.8 & 1.7 & 5.0 & 2.1 \\
\hline 14 & 2.8 & 7.8 & 7.6 & 2.1 & 2.4 & 1.0 & 7.0 & 4.9 & 3.7 & 2.8 & 2.6 & 3.8 \\
\hline 15 & 5.1 & 64.0 & 7.4 & 950.0 & 6.4 & 31.0 & 8.2 & 64.0 & 5.8 & 46.0 & 7.8 & 27.0 \\
\hline 16 & 3.2 & 520.0 & 6.6 & 180.0 & 4.5 & 64.0 & 7.9 & 49.0 & 3.9 & 6.1 & 2.9 & 4.7 \\
\hline 17 & 2.6 & 96.0 & 1.9 & 12.0 & 1.5 & 1.1 & 1.2 & 51.0 & 6.9 & 9.7 & 8.6 & 15.0 \\
\hline 18 & 2.4 & 10.0 & 2.0 & 14.0 & 1.2 & 3.5 & 1.4 & 5.4 & 3.6 & $\begin{array}{l}4.7 \\
\end{array}$ & 3.6 & 6.3 \\
\hline
\end{tabular}

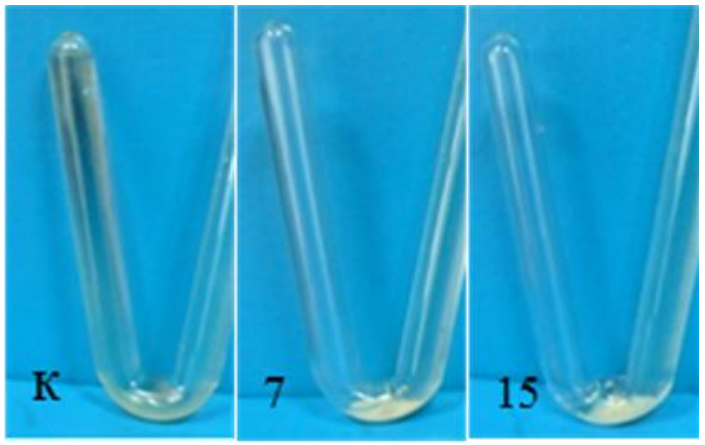

$\mathrm{K}$ - original unadapted production strain No.18.

Figure 3: Fermentation activity of selective yeast variants in the wort with $10 \%$ ethanol

\section{CONCLUSION}

Thus, the consequent adaptation of alcohol yeast to the increased content of sodium chloride in the medium helps to modify their cell walls and increase resistance to the final fermentation product ethanol. Selected variants of alcohol yeast will contribute to the intensification of the process of production of ethyl alcohol and will enhance the profitability of production by increasing the yield of ethanol at the same cost.

\section{REFERENCES}

[1] M. Lopes, L. Serrano, I. Ribeiro, P. Cascão, N. Pires, S. Rafael, L. Tarelho, A. Monteiro, T. Nunes, M. Evtyugina, O.J. Nielsen, S.M. Gameiro, A.I. Miranda, and C. Borrego, Emissions characterization from EURO 5 diese/biodiesel passenger car operating under the new European driving cycle, Atmospheric Environment, 84, 2014, 339-348.

[2] A. Ajanovic and R. Haas, On the future prospects and limits of biofuels in Brazil, the US and EU, Applied Energy, 135, 2014, 730-737.

[3] A.G. Sato, C.D. Silva, V.A. Paganin, L.G. Biancolli, and A.E. Ticianelli, New, efficient and viable system for ethanol fuel utilization on combined electric/internal combustion engine vehicles, Journal of
Power Sources, 294, 2015, 569-573.

[4] J. Baeyens, Q. Kang, L. Appels, R. Dewil, Y. Lv, and T. Tan, Challenges and opportunities in improving the production of bio-ethanol, Progress in Energy and Combustion Science, 47, 2015, 60-88.

[5] N. Kopsahelis, L. Bosnea, A. Bekatorou, C. Tzia, and M. Kanellaki, Alcohol production from sterilized and non-sterilized molasses by Saccharomyces cerevisiae immobilized on brewer's spent grains in two types of continuous bioreactor systems, Biomass Bioenergy, 45, 2012, 87-94.

[6] H. Wu, A. He, X. Kong, M. Jiang, X. Chen, D. Zhu, G. Liu, and W. Jin, Acetonebutanol-ethanol production using $\mathrm{pH}$ control strategy and immobilized cells in an integrated fermentation-pervaporation process, Process Biochemistry, 50, 2015, 614-622.

[7] T.H. Tran, N.N.T. Neil, M.M. Bilek, and D.R. McKenzie, Covalent immobilization of enzymes and yeast: Towards a continuous simultaneous saccharification and fermentation process for cellulosic ethanol, Biomass Bioenergy, 81, 2015, 234241.

[8] M.M. El-Dalatony, M.B. Kurade, R.A.I. Abou-Shanab, K. Hoo, S. El-Sayed, and B.H. Jeon, Long-term production of 
bioethanol in repeated-batch fermentation of microalgal biomass using immobilized Saccharomyces cerevisiae, Bioresource Technology, 219, 2016, 98-105.

[9] L. Mulko, C.R. Rivarola, C.A. Barbero, and D.F. Acevedo, Bioethanol production by reusable Saccharomyces cerevisiae immobilized in a macroporous monolithic hydrogel matrices, Journal of Biotechnology, 233, 2016, 56-65.

[10] X.Q. Zhao, and F.W. Bai, Mechanisms of yeast stress tolerance and its manipulation for efficient fuel ethanol production, Journal of Biotechnology, 144, 2009, 2328.

[11] D.Q. Zheng, X.C. Wu, X.L. Tao, P.M. Wang, P. Li, X.Q. Chi, Y.D. Li, Q.F. Yan, and Y.H. Zhao, Screening and construction of Saccharomyces cerevisiae strains with improved multi-tolerance and bioethanol fermentation performance, Bioresource Technology, 102, 2011, 3020-3027.

[12] M. Zhang, R. Zhu, Zh. Minfeng, and Sh. Wang, Creation of an ethanoltolerant Saccharomyces cerevisiae strain by $266 \mathrm{~nm}$ laser radiation and repetitive cultivation, Journal of Bioscience and Bioengineering, 118, 2014, 508-513.

[13] S.-J. Dong, C.-F. Yi, and H. Li, Changes of Saccharomyces cerevisiae cell membrane components and promotion to ethanol tolerance during the bioethanol fermentation, The International Journal of Biochemistry \& Cell Biology, 69, 2015, 196-203.

[14] E. Ohta, Y. Nakayama, Y. Mukai, T. Bamba, and E. Fukusaki, Metabolomic approach for improving ethanol stress tolerance in Saccharomyces cerevisiae, Journal Bioscience and Bioengineering, 121, 2016, 399-405.

[15] Hohmann S., Osmotic Stress Signaling and Osmoadaptation in Yeasts, Microbiology and Molecular Biology Reviews, 66, 2002, 300-372.

[16] D. A. Los, and_N. Murata, Membrane fluidity and its roles in the perception of environmental signals, Biochimica and Biophysica Acta (BBA)-Biomembranes, 1666 (1-2), 2004, 142-157.

[17] H. Le Gall, F. Philippe, J.-M. Domon, F. Gillet, J. Pelloux, and C. Rayon, Cell Wall Metabolism in Response to Abiotic Stress, Plants, 4 (1), 2015, 112-166. 